\title{
The new gamma-ray observatory: CTA
}

\author{
John Carr for the CTA Collaboration \\ Aix Marseille Université, CNRS/IN2P3, CPPM UMR 7346, 13288 Marseille, France
}

\begin{abstract}
CTA is the next generation gamma-ray observatory and will have a factor 10 better sensitivity compared to existing facilities, as well as many other superior parameters. Aspects of array layout, performance and sites are presented. The broad range of forefront science which will be studied is described.
\end{abstract}

\section{Introduction}

The Cherenkov Telescope Array (CTA) is global effort, with $>1000$ participating scientists and engineers from 28 countries, to deliver an observatory for photon astronomy at the highest energies $(\sim 20 \mathrm{GeV}$ to $\sim 300 \mathrm{TeV})$. CTA will dramatically improve on all aspects of performance with respect to the highly successful current generation of Cherenkov telescope arrays (see e.g. [1]). CTA will survey the sky $\sim 200$ times faster than any current instrument in the energy range above $100 \mathrm{GeV}$, have the best energy flux sensitivity and angular resolution of any instrument operating above the X-ray band and will outperform the Fermi space telescope by 4-5 orders of magnitude in sensitivity for sub-minute-timescale transient phenomena such as gamma-ray bursts. The project combines guaranteed scientific return, in the form of precision very-high-energy astrophysics, with considerable potential for major particle astrophysics discoveries. For example, CTA will conduct searches for dark matter annihilation, which complement direct detection and production (LHC) experiments, and test for Lorentz invariance violation at the Planck scale. The astrophysics of CTA includes new probes of extreme astrophysical environments, such as the neighbourhoods of black holes and neutron stars, relativistic jets, outflows and explosions, and a census of particle acceleration in the universe and the impact of cosmic ray feedback on all scales.

High energy gamma-rays offer the only sensitive probe of the ultra-relativistic protons and nuclei which may dominate the pressure in environments from supernova remnant shells to cluster-scale active galactic nucleus blown bubbles. The breadth and depth of the CTA science case has made it a key project in the field of particle astrophysics. CTA will be the first particle astrophysics instrument to be operated as an open observatory, serving a wide scientific community. The science of CTA is described in detail in a recent special issue of the journal Astroparticle Physics [2].

The technologies planned for CTA represent an evolution of those employed in the existing imaging atmospheric Cherenkov telescopes H.E.S.S [3], MAGIC [4] and VERITAS [5]. The key hardware elements are telescope structures, cameras, mirrors, and data-processing infrastructure. The concept for telescope structure is similar to that of MAGIC in the case of the LST, and the MST structure combines elements of the VERITAS and HESS designs. Camera technologies are considerably improved with respect to current instruments, with multiple viable technology options in some cases. Mirror technologies are likewise significantly improved. 
The CTA Observatory will be located at two sites, one in the southern and one in the northern hemisphere, at each site combining Cherenkov telescopes of different sizes and designs to cost-effectively cover the very wide energy band. The sites will complement each other, providing full-sky coverage for Galactic and extragalactic sources. While the southern site, with privileged Milky Way views, will have energy coverage up to $300 \mathrm{TeV}$, allowing exploration of Galactic sources up to the highest energies, the northern site will have more limited high-energy capabilities.

At least three telescope types are required to cover the full CTA energy range in a costeffective way: small, medium and large sized telescopes. An extension for the southern observatory is envisaged based on a fourth, medium-sized Schwarzschild Couder telescope type. The expected performance of CTA performance has been established using very detailed simulations, which have been verified by comparison to existing instruments. The required performance can be achieved at all CTA candidate sites investigated and for a range of implementation options, being rather insensitive to details of the telescope layout. Science performance has been demonstrated through a number of detailed case studies.

\section{CTA arrays}

To achieve full sky coverage, the CTA Observatory will be located on two sites, one in the southern and one in the northern hemisphere. Each telescope consists of a primary reflector and in some cases a secondary reflector, a camera and a drive system. Telescope control, array-level event-selection/triggering and data processing will be handled by centralised systems. The two sites will have different number and layout of telescopes. To cover a wide energy range in a cost-effective way, the arrays will contain several different types of telescope:

- The low energy instrumentation, efficiently measuring gamma-rays with energies below a few hundred GeV, will consist of 23 metre diameter, Large-Sized Telescopes (LSTs) with a field of view (FoV) diameter of at least $4.4^{\circ}$.

- The medium energy range, from around $100 \mathrm{GeV}$ to more than $10 \mathrm{TeV}$, will be covered by 12 metre diameter Medium-Sized Telescopes (MSTs) with a FoV of at least $7^{\circ}$. The Schwarzschild-Couder Telescope (SCT) is a second type of medium-sized telescope, using a two-mirror design, which will be used to extend the area covered by mediumsized telescopes beyond the baseline configuration to more than $1 \mathrm{~km}^{2}$ and improve angular resolution.

- The high energy instrumentation, mainly operating above $10 \mathrm{TeV}$, will consist of a large number of 4 metre diameter, Small-Sized Telescopes (SSTs) with a FoV of at least $8^{\circ}$.

The baseline layout of the various telescopes on the two sites is shown in Fig. 1, and schematics of the main dimensions of the different telescopes are shown in Fig. 2. Figure 1 includes the SCTs, extending the baseline MST array. Depending on the deployment timescales of the baseline MST telescopes, and of the additional SCTs, a "hybrid" configuration may be employed where MSTs and SCTs are interleaved.

\section{Performance}

The contribution of the different types of telescopes to the overall sensitivity for on-axis point sources is demonstrated for an example array layout in Fig. 3, for an observation time of 50 hours. The cross-over in sensitivity between the LST and MST components occurs at about $250 \mathrm{GeV}$ and that between MSTs and SSTs at about $4 \mathrm{TeV}$. At the LST/MST as well as the MST/SST transition, the combined sensitivity is almost a factor of two better than 


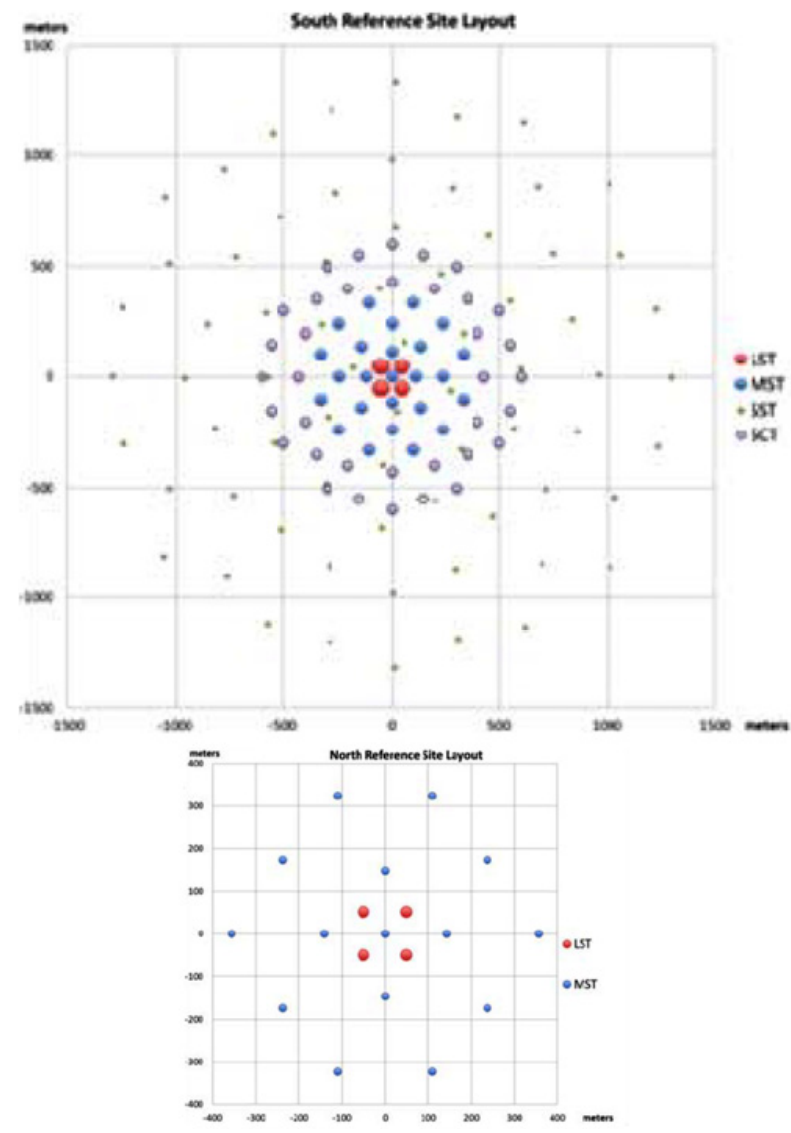

Figure 1. Example layouts of telescopes on the two sites in the southern hemisphere (top) and northern hemisphere (bottom) with different scales. The symbols indicate the locations of the telescopes as: red LST; blue MST; open purple SCT and green for SST. The southern site contains more telescopes and covers a larger area.

that of the individual components. However, close to $1 \mathrm{TeV}$, the onset of low-quality (close to threshold) SST data, with large effective area but poor gamma-hadron rejection can reduce the combined sensitivity in our simplest analysis method to close to the sensitivity of MST data alone. With the smaller SSTs in current designs, the MST/SST cross-over point is expected to occur at higher energies, but still below $10 \mathrm{TeV}$.

The expected CTA sensitivity is compared to that of other observatories in Fig. 5.

\section{Candidate sites}

Following a call for site proposals, a total of nine proposal documents were received with sites in several countries in the world. The map in Fig. 5 indicates the locations of all sites considered.

Among the candidate sites proposed, the one at Sutherland in South Africa was withdrawn by the proposers. The sites in India and China were considered low priority for study and have not been evaluated in detail.

The site in Spain is located $5 \mathrm{~km}$ from the Teide Observatory in Tenerife. The site in Mexico is $5 \mathrm{~km}$ from the San Pedro Martir Observatory in Baja California Mexico. The two 


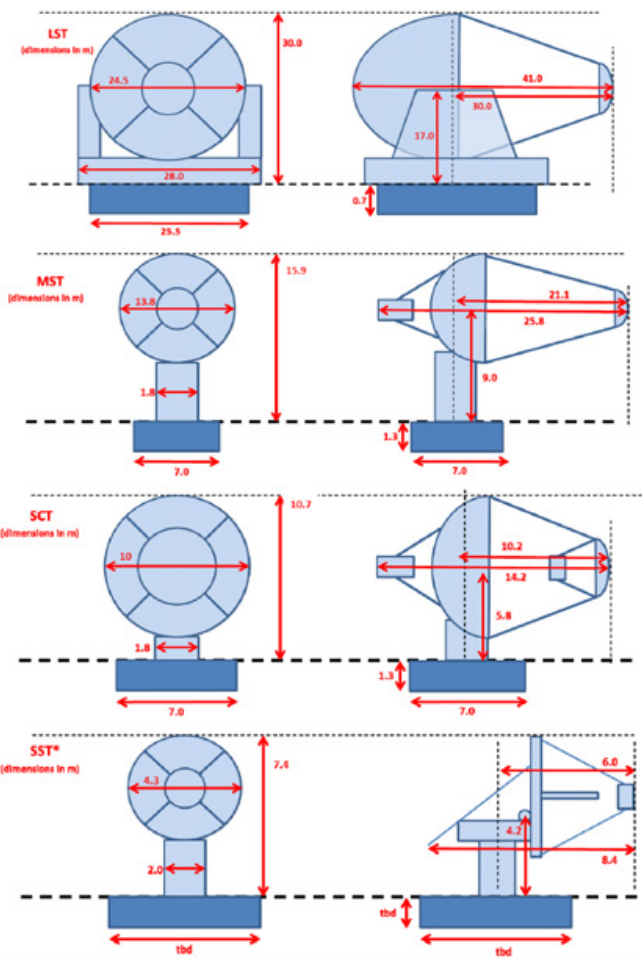

Figure 2. Indicative sketches of the four sizes of telescopes.

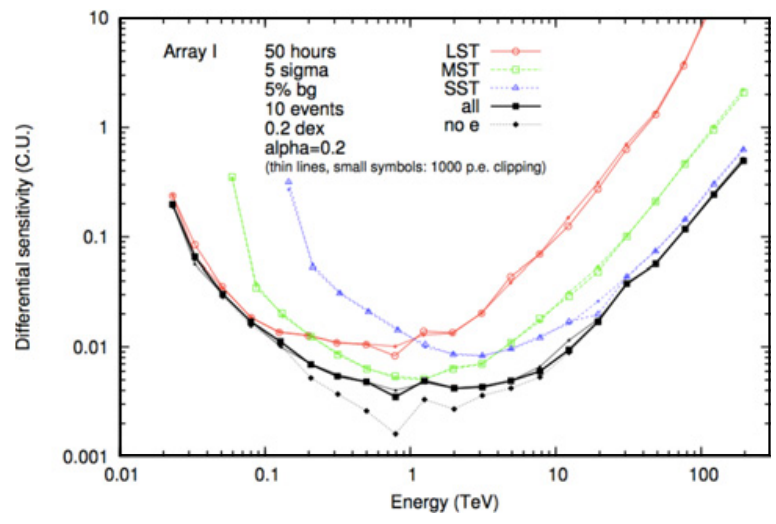

Figure 3. Point source sensitivity of for an example array layout (solid black line, filled squares) compared to that of its component sub-systems: 3 LSTs (red, open circles), 18 MSTs (green, open squares), and 56 SSTs (blue, open triangles). The importance of the background of cosmic ray electrons for the combined sensitivity is demonstrated by the dashed line with diamonds, where this background is ignored.

sites in the USA are located in Arizona Yavapai $160 \mathrm{~km}$ west and Meteor Crater $70 \mathrm{~km}$ east of Flagstaff where the Lowell Observatory is located. In Argentina the Leoncito site is located within the CASLEO reserve in San Juan province and the San Antonio site is located near the city of San Antonio de los Cobres in Salta province. In Namibia, one site is adjacent to the location of the HESS Gamma-ray Telescope array, roughly 100km south of the capital 


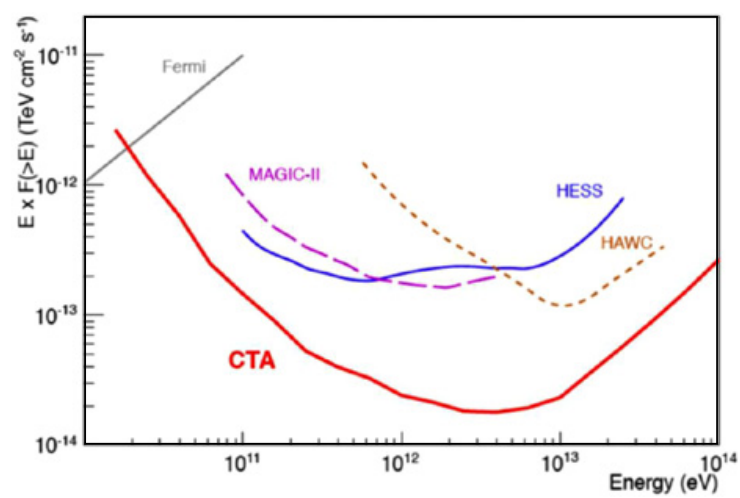

Figure 4. Integral sensitivity for CTA from MC simulations, together with the sensitivities in comparable conditions ( $50 \mathrm{~h}$ for IACTs, 1 year for Fermi-LAT and HAWC) for some gamma-ray observatories.

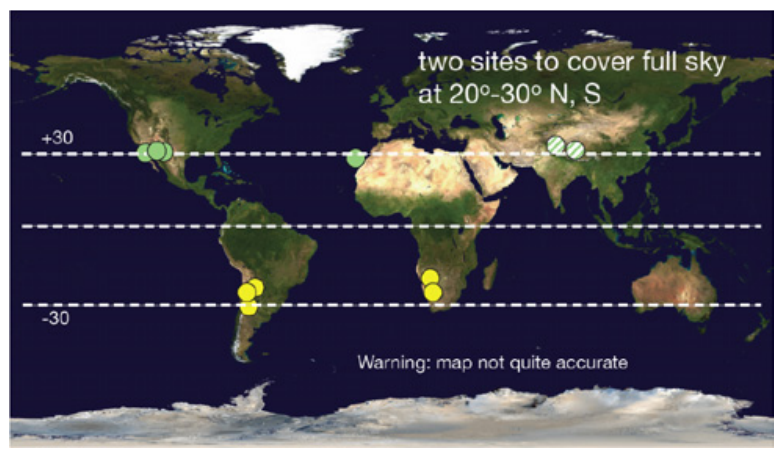

Figure 5. Approximate locations of all site candidates proposed.

city of Windhoek and another site is between Keetmanshoop and Lüderitz, about $20 \mathrm{~km}$ from the town of Aus. In Chile, the site is close to the Paranal Observatory about $120 \mathrm{~km}$ south of Antofagasta.

Following detailed studies of the candidate sites a ranking was made by the collaboration. With advice from an independent committee, the CTA funding agencies choices the sites in Chile and Namibia as the preferred locations for the Southern site and negotiations have started to make the final site choice in this hemisphere by summer 2015. A similar process will take place for the Northern hemisphere during 2015.

\section{Science}

Ground-based gamma-ray astronomy is a young field with enormous scientific potential. The possibility of astrophysical measurements at teraelectronvolt $(\mathrm{TeV})$ energies was demonstrated in 1989 with the detection of a clear signal from the Crab (Pulsar Wind) Nebula above $1 \mathrm{TeV}$ with the Whipple Imaging Atmospheric Cherenkov Telescope (IACT). Since then, the instrumentation for, and techniques of, astronomy with IACTs have evolved to the extent that a flourishing new scientific discipline has been established, with the detection of 150 sources and a major impact in astrophysics and more widely in physics. The current major arrays of IACTs: HESS, MAGIC, and VERITAS, have demonstrated the huge physics potential at these energies as well as the maturity of the detection technique. Many 
astrophysical source classes have been established, some with many well-studied individual objects, but there are indications that the known sources represent the tip of the iceberg in terms of both individual objects and source classes. The Cherenkov Telescope Array will transform our understanding of the high-energy universe. An advanced member of the suite of forthcoming major astroparticle physics experiments and observatories; CTA will exploit synergies with projects seeking gravitational wave and astrophysical neutrino detection as well as with classical photon observatories. The synergy with the upcoming Square Kilometre Array (SKA) is perhaps the most exciting in terms of mutual scientific benefit. CTA will address a wide range of major questions in and beyond astrophysics, which can be grouped in to three broad themes:

\section{Theme 1: Understanding the Origin and Role of Relativistic Cosmic Particles}

- What are the mechanisms for cosmic particle acceleration?

- What are the sites of high-energy particle acceleration in the universe?

- What role do accelerated particles play in feedback on star formation and galaxy evolution?

\section{Theme 2: Probing Extreme Environments}

- What physical processes are at work close to neutron stars and black holes?

- What are the characteristics of relativistic jets, winds and explosions?

- How intense are radiation fields and magnetic fields in cosmic voids, and how do these evolve over cosmic time?

\section{Theme 3: Exploring Frontiers in Physics}

- What is the nature of Dark Matter? How is it distributed?

- Are there quantum gravitational effects on photon propagation?

- Do axion-like particles exist?

\subsection{Understanding the origin and role of relativistic cosmic particles}

Relativistic particles appear to play a major role in a very wide range of astrophysical systems, from supernova explosions to the galaxy cluster-scale outbursts of active galactic nuclei. Within the interstellar medium of our own galaxy these cosmic rays are close to pressure equilibrium with turbulent motions of gas and magnetic fields, yet the relationship between these three components, and the overall impact on the star-formation process and the evolution of galaxies, is very poorly understood. CTA will provide the first real precision measurements of cosmic ray protons and nuclei (rather than the energetically sub-dominant electrons that produce the non-thermal emission seen at radio and X-ray wavelengths) in astrophysical systems, providing insights in to the processes of acceleration, transport and the cosmic ray mode feedback mechanisms in these systems. Historically, non-thermal effects in astrophysical systems have largely been ignored or parameterized away due to a lack of high quality data. The insights from CTA will represent a major contribution to our deepening understand of the processes by which galaxies and clusters of galaxies evolve.

\section{Cosmic accelerators}

The primary goal of gamma-ray astrophysics thus far has been to establish in which cosmic sources particle acceleration takes place, and in particular, to establish the dominant contributors to the locally measured cosmic rays which are $99 \%$ protons and nuclei (usually hadrons). Huge progress has been made in the last decade in this area, with the combination of Fermi-LAT and IACT data proving extremely effective in identifying the brightest Galactic accelerators and providing strong evidence of hadron acceleration in a handful of sources. However, key questions remain unanswered: are supernova remnants (SNR) the only major 
contributor to the Galactic cosmic rays? Where in our galaxy are particles accelerated up to $\mathrm{PeV}$ energies? What are the sources of high-energy cosmic electrons? What are the sources of the ultra-high energy cosmic rays? CTA will address all of these questions and also the critical issue of the mechanisms for particle acceleration at work in cosmic sources, through two main approaches:

- A census of particle acceleration in the universe, with Galactic and Extragalactic surveys and deep observations of key nearby galaxies and clusters.

- Precision measurements of archetypal sources, where bright nearby sources will be targeted to provide resolved spectroscopy or very high statistics light curves to provide a deeper physical understanding of the processes at work in cosmic accelerators.

A general census is required to understand the populations of accelerators, and the evolution/life-cycle of these source classes. Deep observations of individual sources are required to acquire the very broad band spectra needed to unambiguously separate lepton and hadron acceleration and to test acceleration to the highest energies possible for Galactic accelerators. A key target of CTA will be the bright, young supernova remnant (SNR) RX J1713-3946 where the dominant gamma-ray emission mechanism is unclear from current measurements, but where CTA can resolve the ambiguity and resolve sub-structure within the SNR shell. Known TeV-emitting source classes where CTA data will have a transformational impact on our understanding include pulsar wind nebulae, gamma-ray binaries, collidingwind binaries, massive stellar clusters, starburst galaxies and active galaxies. There is clearly huge potential for the discovery of new classes of accelerator, with emission on the scale of clusters of galaxies as one of the most exciting possibilities.

\section{Propagation and influence of accelerated particles}

Beyond the question of how and where particles are accelerated in the universe, is the question of what role these particles play in the evolution of their host objects and how they are transported up to larger and larger scales. On the scale of clusters of galaxies, cosmic rays with $\mathrm{TeV}-\mathrm{PeV}$ energies are thought to be confined over a Hubble time, on smaller scales they typically escape from their acceleration sites and may impact upon their environments in a number of ways:

1. As a dynamical constituent of the medium

2. Through generation / amplification of magnetic fields

3. Through ionization and subsequent impact on the chemical evolution of (for example) dense cloud cores.

All these effects are relevant for the interstellar medium of our own galaxy, and are likely to be important in star-forming systems on all scales and also for the process of AGN feedback on the host galaxy cluster and growth of massive galaxies.

CTA will map extended emission around many gamma-ray sources and look for energy dependent morphology associated with diffusion (in the case of hadrons) or cooling (in the case of electrons). As the energy-dependence is expected to be opposite in the two cases, such mapping provides another means to separate emission from these two populations. It is CTAs unprecedented angular resolution, energy resolution and background rejection power that make this possible.

\subsection{Probing extreme environments}

Particle acceleration to very high energies is typically associated with extreme environments: close to compact objects such as neutron stars and black holes, or in relativistic outflows 
or explosions. VHE emission from accelerated particles can therefore act as a probe of these environments, providing access to time and distance scales which are inaccessible in other wavebands. VHE emission escapes from systems where UV and X-ray emission is absorbed, and provides information independent of assumptions on magnetic field strengths. In addition, VHE photons from distant objects can be used as a probe of the intervening space. Gamma-gamma pair production will allow us to measure the redshift evolution of the UV-IR background and hence the star-formation history of the universe, and probe magnetic fields in cosmic voids down to values many orders of magnitude below the reach of any other technique. CTA will establish if the VHE photons heat the gas in these under dense regions, suppressing the formation of dwarf satellite galaxies.

\section{Black holes and jets}

Active galactic nuclei are thought to harbour supermassive black holes (SMBHs), accreting material and producing collimated relativistic outflows by a still poorly-understood process. Similarly, accreting stellar mass black holes are known to produce jets and particle acceleration seems to be universally associated with BH-powered jets. Acceleration may occur extremely close to the SMBH (in the case of very low accretion rate systems) up to Mpc scales where the largest AGN jets finally terminate. Active galaxies are seen as one of the most likely sites of the acceleration of the ultra-high energy cosmic rays, with energies up to energies around $10^{20} \mathrm{eV}$, but so far there is an absence of any strong evidence for hadronic acceleration in AGN jets. Simultaneous broad-band data is needed to study variable jet emission in both galactic and extragalactic systems, with CTA data playing a key role: establishing the presence of very high energy particles, identifying the presence of hadrons and probing extremely short timescale variability that provides information on the smallest scales, probing the bulk ultra-relativistic motions of the inner jet. Time-resolved VHE spectral measurements are key to disentangling leptonic and hadronic emission scenarios, and to study jet power and dynamics, and probe magnetic fields in this extreme environment. The low luminosity AGN in the heart of our own galaxy, Sgr A*, is coincident with a $\mathrm{TeV}$ source and has associated non-thermal emission in the radio and X-ray. However, the sensitivity, resolution and pointing precision of current gamma-ray telescopes is insufficient to separate emission from very nearby sources and the diffuse emission around the Galactic Centre. CTA will map this region in unprecedented detail, probing the relationship between the central source and the diffuse emission, and on much larger scales, to the Fermi bubbles. There is evidence from current IACTs for TeV emission from a single system hosting a stellar mass black hole: Cygnus X-1. The sensitivity of CTA should allow this object to be studied in detail and provide new detections, opening the door to studies of high-energy non-thermal processes associated with stellar mass black holes and allow the first comparisons to be made with SMBH systems.

\section{Neutron stars and relativistic outflows}

CTA will probe the environment around neutron stars via pulsed gamma-ray emission from the magnetosphere of pulsars, and the ultra-relativistic outflows of these systems via mapping and spectral measurements of the associated synchrotron/IC nebula and (possibly and uniquely) the un-shocked pulsar wind. Young and energetic (in terms of available rotational energy) pulsars cluster tightly along the Galactic plane and hence the majority of objects will be covered. Two key objects: HESSJ1825-137 and the Vela pulsar (and associated Vela X nebulae), will be studied in the CTA Key Science Programmes. Binary systems including a pulsar provide a unique opportunity to study a relativistic outflow under changing physical 
conditions as the orbit progresses, via energy-dependent light-curve measurements. Merging neutron stars and other compact object mergers are the likely counterparts of short GRBs and are expected to give rise to detectable gravitational wave transients in the near future. CTA will rapidly respond to triggers from gravitational wave or GRB instruments, to probe the highest energy processes associated with such events.

\section{Cosmic voids}

Much of the universe consists of extremely under-dense regions known as cosmic voids. Very high energy photos interact within these voids, and allow us to probe the radiation fields and magnetic fields that they contain. The Extragalactic Background Light (EBL) is the integrated emission from stars and galaxies of all types through the evolution of the universe. As such it is an important tool for cosmology, but is extremely difficult to measure directly, due to very strong foregrounds from the solar system and the Milky Way. However, the EBL leaves an imprint on the measured spectra of gamma-ray sources, via the process of gammagamma pair production. The wide-band, high-quality spectra measured with CTA, for a large number of objects, will allow the EBL spectrum from the optical to the far infrared to be very precisely measured at redshift zero. Furthermore, with the expected large sample of blazars up to redshift $\sim 1$ detected with CTA, the evolution of the EBL with cosmic time can be probed for the first time. The pair-production of $\mathrm{TeV}$ photons in voids also offers the prospect of measuring the extremely weak magnetic fields thought to exist in these regions. Secondary gamma-rays are produced by the original $\mathrm{e}^{ \pm}$pairs via inverse Compton scattering on the EBL. A cascade can then develop from further pair and IC interactions. Depending on the typical value of the intergalactic magnetic fields (IGMF) deflections of the secondary particles may either be small enough that secondary components may be observable as pair echoes, which arrive with a time delay relative to the primary emission, or as a pair halo, potentially resolvable extended emission around the primary source. The properties of the extended emission depend on the IGMF strength. Strong enough IGMF $\left(>10^{-12} \mathrm{G}\right)$ leads to full isotropisation of the cascade emission and formation of a physical pair halo, while weaker magnetic field leads to appearance of an extended emission with an IGMF-dependent size. If the IGMF strength is in the range, $\mathrm{B} \sim 10^{-16}$ to $10^{-12} \mathrm{G}$, the spatially-extended emission may be detectable and resolvable by CTA by virtue of its high sensitivity and angular resolution; e.g., for a source at a distance of $100 \mathrm{Mpc}$, the extended emission would be on a $\sim 1^{\circ}$ scale, and be comfortably contained within the CTA FoV. If, as has been recently suggested, TeV electrons produced in gamma-gamma interactions in the voids do not initiate cascades but rather heat the ultra-low dense plasma, CTA will allow this hypothesis to be proven and the heating rate to be very well constrained. As such heating could be the dominant means of heating in low density regions after redshift $\sim 2$, and could solve the problem of the missing dwarf satellite galaxies, this measurement would be a very valuable addition to cosmology.

\subsection{Physics frontiers}

The reach of CTA encompasses considerable discovery space in the area of fundamental physics. CTA will reach the expected thermal relic cross-section for self-annihilating dark matter for a wide range of dark matter particle masses, including those inaccessible to the LHC. The long travel paths of gamma-rays from extragalactic sources combined with their short wavelength make them a sensitive probe for energy dependent variation of the speed of light due to quantum-gravity induced fluctuations of the metric. CTA will be sensitive to such effects on their expected characteristic scale: the Planck scale. On their long journey, gamma-rays may couple to other light particles such as axions, under the 
influence of intergalactic magnetic fields. Such photon-axion oscillations effectively make the Universe more transparent to gamma-rays, and akin to neutrino oscillations introduce a spectral modulation. Each of these effects would represent a very major discovery, alone worth the effort of constructing and operating CTA. The major step in sensitivity and energy coverage that CTA represents brings such effects within reach, and will undoubtedly allow further issues in fundamental physics to be addressed.

\section{Quantum gravity and axion search}

The beams of photons with energies up to hundreds of $\mathrm{TeV}$, produced by cosmic sources and detected with CTA, represent a powerful tool to explore departures from the Standard Model and search for a wide range of new physics. The two main areas in fundamental physics, beyond the dark matter search are the search for axion-like particles (ALPs) and evidence of Lorentz Invariance Violation (LIV) associated with the quantum nature of gravity. Blazars and gamma-ray bursts are thought to be the most promising (bright, distant) target classes for both these searches. It has been suggested that QG effects may induce time delays between photons with different energies travelling over large distances due to a nontrivial refractive index of the vacuum. High statistics measurements over a very wide energy range of GRBs and Blazars will allow CTA to make order of magnitude better constraints than possible with current IACTs, reaching deep in to the interesting parameter space for models.

Axions are expected to convert into photons (and vice versa) in the presence of magnetic fields. In the case of a very distant AGN, the ALP/photon coupling can result in a detectable enhancement of the TeV photon flux (in competition with the EBL absorption), depending on the ALP mass. CTA ALP limits will complement those from indirect astrophysical tests, from solar observations, and from X-ray telescopes.

\section{Dark Matter}

The nature of dark matter (DM) in the universe is one of the most compelling questions facing physics and astronomy at the present time. There are many programmes around the world that have capabilities or are devoted to searching for non-gravitational signals of DM. Among these, CTA has an almost unique capability during the next decade to explore the WIMP mass region in the many hundreds of $\mathrm{GeV}$ to multi-TeV regime, above the reach of the LHC and ton-scale direct detection experiments. To capitalize on this remarkable opportunity, it is imperative for CTA to devote substantial resources (both observing time and effort) towards a comprehensive and integrated DM program. If this is done, CTA will be a cornerstone of a global, multi-faceted, line of attack from different experiments probing with different methods to understand the nature of the dominant gravitational matter in the Universe.

The indirect DM search with CTA has several possible astrophysical targets each of them with its own inherent advantages and disadvantages. The Milky Way (MW) galaxy represents a natural place to look for DM signatures and its centre is expected to be the brightest source in the DM induced gamma-ray sky, although the exact magnitude is rather uncertain. The DM density profile in the MW should also lead to an annihilation signal all around us; however astrophysical galactic foregrounds coupled with the enormous spatial extent and the truly diffuse nature of this galactic DM emission make separation between signal and background problematic. On the other hand, nearby dwarf spheroidal galaxies should provide easier separation of signal and background but comparatively lower signals because of both the distance and lower DM content compared to the Milky Way, even though somewhat compensated by higher DM concentrations. 
The concordance cosmological CDM model predicts that the formation of visible structures has been guided by gravitational accretion of baryons onto previously formed DM over-densities. The astrophysical structures of interest result from the hierarchical formation of DM halos from primordial DM over-densities. The subsequent evolution of these DM halos occurred in many different ways. In particular on galactic and subgalactic scales, the process depends on halo parameters such as the halo mass and the mass density profile, the evolution history, and conditions set by the local galactic evolution environment.

Some of the resulting halos could have been sufficiently massive to accrete enough baryons to initiate star formation and give origin to galaxies, including the variety of satellite galaxies we actually observe in the Milky Way halo. In-falling dwarf galaxies (e.g., dwarf irregular galaxies) approaching more central parts of their host halo could have evolved to dwarf spheroidal galaxies (dSphs). These dwarf galaxies, being highly DM dominated and comparatively close by, form one of the primary targets for CTA observations of this proposal and have already been subject to extensive studies with currently operating IACTs. However, for various reasons including the halo size, location, encounters, the baryonic content of the environment, or the presence of a central Black Hole, less massive DM clumps could have evolved differently and not into a visible $\mathrm{dSph}$. They would constitute DM-dominated overdensities purely observable in gamma-rays or cosmic-rays emerging from DM annihilations. While, in principle, secondary emission from inverse Compton or synchrotron scattering processes (dependent on ambient photon and magnetic fields, respectively) will lead to diffuse multi-wavelength (MWL) signatures, classical MWL survey techniques do not provide sufficient sensitivity for detecting their signatures. Such objects are commonly known as DM subhalos or DM clumps.

The centre of the Milky Way galaxy has in the past been considered as a target for DM searches but more recently, because of the rich field of VHE gamma-ray astrophysical sources in the region, the searches focus on the galactic halo excluding the central region of galactic latitude $b<0.3^{\circ}$. Even excluding the very central region, the total mass of DM in the galactic halo together with its proximity to Earth make it the most promising source for DM searches in CTA. The inconvenience of this target, however, is the fact that being a diffuse source, the integration over the inner halo, while yielding a large signal, gives a very large instrumental background from misidentified charged cosmic rays. Further, there are astrophysical backgrounds from various sources which must be understood, even with the very central region excluded from the analysis.

It is believed that the disadvantages of the MW target can be overcome with sufficient experimental effort to control systematic effects in background subtraction.

The Large Magellanic Cloud is a massive nearby satellite galaxy visible from the southern hemisphere, at Galactic latitude of $-32.9^{\circ}$. It is located at a distance $d=50 \mathrm{kpc}$ and it has the shape of a disk seen nearly face-on. The LMC hosts many interesting astrophysical sources: the largest star-forming region of the local group of galaxies; one of the densest stellar clusters ever known; the most massive stars ever observed; several tens of HII regions; more than a dozen super-bubbles; numerous giant shells; more than 50 catalogued SNRs and the remnant of the recent naked-eye supernova SN 1987A. For the search for DM, the mass of the LMC which is $1 \%$ of the MW makes it interesting even when its distance at $\times 6$ further away than the Galactic Centre gives a large signal reduction. Although smaller in subtended angle than the MW, with most of the emission within the CTA FoV, the LMC is also an extended source where the backgrounds are again a significant drawback.

The dwarf spheroidal galaxies (dSphs) of the Local Group could give a clear and unambiguous detection of DM. They are gravitationally bound objects and are believed to 


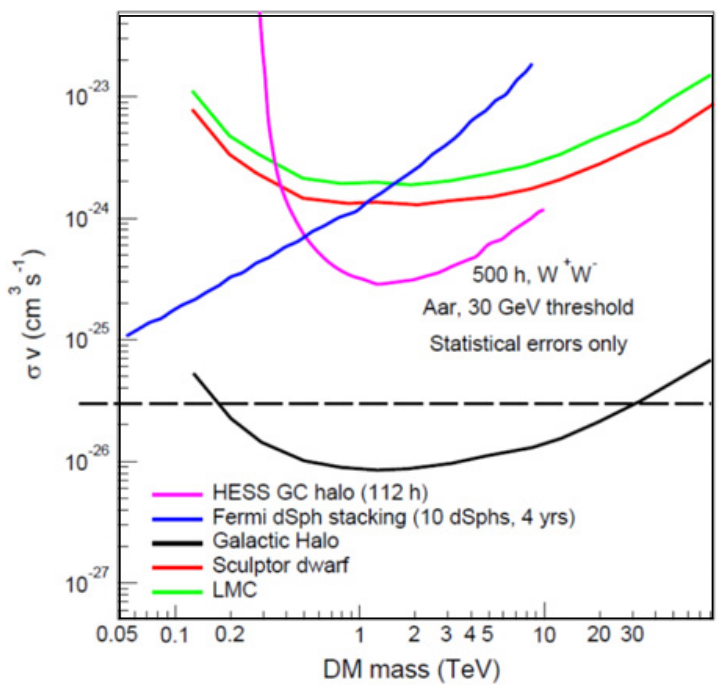

Figure 6. Comparison of predicted sensitivities in $\left\langle\sigma_{\chi \chi} \mathrm{v}\right\rangle$ for the targets of: the Milky Way Halo; the Large Magellanic Cloud and the dwarf galaxy Sculptor. The CTA sensitivity curves use the same method and $\mathrm{W}^{+} \mathrm{W}^{-}$annihilation modes for each target and the NFW DM profile. The sensitivities for the three targets are all for 500 hours taking into account only statistics errors; for the MW and the LMC, the systematics of backgrounds must be very well controlled to achieve this statistically possible sensitivity. The comparisons with the HESS and Fermi-LAT results respectively with the bb annihilation modes as published.

contain up to $\mathrm{O}\left(10^{3}\right)$ times more mass in DM than in visible matter, making them widely discussed as potential targets.

Being small and distant many of the dwarf galaxies will appear as near point sources in CTA and hence the nuisance of the instrumental background is much reduced. Further, they are also environments with a favourably low astrophysical gamma-ray background. Neither astrophysical gamma-ray sources (SNRs, pulsar wind nebulae, etc.) nor gas acting as target material for cosmic rays, have been observed in these systems.

Structure formation predicts gravitationally bound DM clumps down to much lower masses than observed for dSph galaxies. The low-mass cut-off of the clump distribution is related to the free-streaming scale of DM particles in the early universe and is expected between $10^{-12} \mathrm{M}_{\text {sun }}$ and $10^{-3} \mathrm{M}_{\text {sun }}$ for typical WIMP scenario. The number-count distribution of clumps in MW-like galaxies has been investigated with numerical N-body simulations, finding a power law: $\mathrm{dN} / \mathrm{dM} \propto \mathrm{M}^{-\alpha}$ in clump mass $\mathrm{M}$, with a high-mass cutoff at $\mathrm{M} \sim 10^{10} \mathrm{M}_{\text {sun }}$ and an index $\alpha$ being between 1.9 and 2.0. The total number of clumps orbiting within galactic halos can be as high as $10^{15}$. For the MW this would imply a clumpiness factor of $\sim 18 \%$ (referring to the relative contribution of the total mass in clumps to the total mass of the main halo). The spatial distribution of DM clumps is biased away from the smooth central DM distribution of the host halo, i.e., the majority of clumps populate outer halo regions $(\mathrm{r}>100 \mathrm{kpc})$.

The subhalo population above $10^{5} \mathrm{M}_{\text {sun }}$ can be probed using gravitational millilensing of background objects while the statistical properties of lighter clumps in the solar neighbourhood can be measured from gravitational nano-lensing.

The Galactic Centre (GC) region is the prime target to look for DM due to its proximity and the high concentration of DM. The DM distribution in the halo centre inferred from Nbody simulations without baryons follows a cuspy profile. However the place where the DM 
annihilation signal is expected to be the strongest is also the place where the baryons settle and perhaps dominate the overall gravitational potential well. Even if there is no consensus yet on how baryons affect the DM distribution, the GC is expected to be the brightest source of DM annihilations in the gamma-ray sky. However, the GC is a crowded region with several known high-energy gamma-ray emitters and more are expected to be detected thanks to the CTA observational capabilities. Given the standard astrophysical emission in this region, the quest for DM requires a deep and uniform exposure over several degrees around the central black hole Sgr A* to allow for both spectral and spatial morphological studies, a deep understanding of the instrumental and observational systematics, and precise measurements of the standard astrophysical emissions. The expected CTA angular and energy resolutions are key ingredients to disentangle a DM signal from standard astrophysical background contamination.

The sensitivity predictions for the Galactic Halo, the dwarf galaxy Sculptor and the Large Magellanic Cloud are compared in Fig. 6. Here it can be clearly seen that the sensitivity possible with the Galactic Halo observation is very much better than that which is possible with a single dwarf galaxy and the LMC. Also it can be seen that the sensitivity possible with Sculptor for $500 \mathrm{~h}$ is better than that for the LMC. Although this plot does not show the effect of systematics, the relative rankings of the sensitivities is not changed by including them.

\section{Summary}

The design of CTA telescope and the site selection process is well advanced. It is expected that founding agreements will be signed during 2015 so construction can start soon afterwards.

We gratefully acknowledge financial support from the following agencies and organizations: Ministerio de Ciencia, Tecnología e Innovación Productiva (MinCyT), Comisión Nacional de Energía Atómica (CNEA), Consejo Nacional de Investigaciones Científicas y Técnicas (CONICET), Argentina; State Committee of Science of Armenia, Armenia; Conselho Nacional de Desenvolvimento Científico e Tecnológico (CNPq), Fundacão de Amparoà Pesquisa do Estado do Rio de Janeiro (FAPERJ), Fundacão de Amparo à Pesquisa do Estado de São Paulo (FAPESP), Brasil; Croatian Science Foundation, Croatia; Ministry of Education, Youth and Sports, MEYS LE13012, 7AMB12AR013, Czech Republic; Ministry of Higher Education and Research, CNRS-INSU and CNRS-IN2P3, CEA-Irfu, ANR, Regional Council Ile de France, Labex ENIGMASS, OSUG2020 and OCEVU, France; Max Planck Society, BMBF, DESY, Helmholtz Association, Germany; Department of Atomic Energy, Department of Science and Technology, India; Istituto Nazionale di Astrofisica (INAF), MIUR, Italy; ICRR, University of Tokyo, JSPS, Japan; Netherlands Research School for Astronomy (NOVA), Netherlands Organization for Scientific Research (NWO), Netherlands; The Bergen Research Foundation, Norway; Ministry of Science and Higher Education, the National Centre for Research and Development and the National Science Centre, Poland; MINECO support through the National R+D+I, CDTI funding plans and the CPAN and MultiDark Consolider-Ingenio 2010 programme, Spain; Swedish Research Council, Royal Swedish Academy of Sciences, Sweden; Swiss National Science Foundation (SNSF), Ernest Boninchi Foundation, Switzerland; Durham University, Leverhulme Trust, Liverpool University, University of Leicester, University of Oxford, Royal Society, Science and Technologies Facilities Council, UK; U.S. National Science Foundation, U.S. Department of Energy, Argonne National Laboratory, Barnard College, University of California, University of Chicago, Columbia University, Georgia Institute of Technology, Institute for Nuclear and Particle Astrophysics (INPAC-MRPI program), Iowa State University, Washington University McDonnell Center for the Space Sciences, USA. The research leading to these results has received funding from the European Union's Seventh Framework Programme (FP7/2007-2013) under grant agreement $n^{\circ} 262053$. 


\section{References}

[1] Hinton \& Hofmann (2010), Teraelectronvolt Astronomy, Ann. Rev. Astron. Astrophys. 47, 523

[2] Seeing the High-Energy Universe with the Cherenkov Telescope Array, Astroparticle Physics 43, Hinton, Sarkar, Torres \& Knapp (2013)

[3] HESS website: http://www.mpi-hd.mpg.de/hfm/HESS

[4] MAGIC website: http://magic.mppmu.mpg.de/

[5] VERITAS website: http://veritas.sao.arizona.edu/ 